\title{
Decay Lengths for Diffusive Transport Activated by Andreev Reflections in Al/n- GaAs/AI Superconductor-Semiconductor-Superconductor Junctions
}

Kutchinsky, Jonatan; Taboryski, Rafael Jozef; Clausen, Thomas; Sørensen, Claus Birger; Kristensen, Anders; Lindelof, Poul Erik; Hansen, Jørn Bindslev; Jacobsen, Claus Schelde; Skov, Johannes

Published in:

Physical Review Letters

Link to article, DOI:

10.1103/PhysRevLett.78.931

Publication date:

1997

Document Version

Publisher's PDF, also known as Version of record

Link back to DTU Orbit

Citation (APA):

Kutchinsky, J., Taboryski, R. J., Clausen, T., Sørensen, C. B., Kristensen, A., Lindelof, P. E., Hansen, J. B., Jacobsen, C. S., \& Skov, J. (1997). Decay Lengths for Diffusive Transport Activated by Andreev Reflections in Al/n-GaAs/Al Superconductor-Semiconductor-Superconductor Junctions. Physical Review Letters, 78(5), 931934. https://doi.org/10.1103/PhysRevLett.78.931

\section{General rights}

Copyright and moral rights for the publications made accessible in the public portal are retained by the authors and/or other copyright owners and it is a condition of accessing publications that users recognise and abide by the legal requirements associated with these rights.

- Users may download and print one copy of any publication from the public portal for the purpose of private study or research.

- You may not further distribute the material or use it for any profit-making activity or commercial gain

- You may freely distribute the URL identifying the publication in the public portal 


\title{
Decay Lengths for Diffusive Transport Activated by Andreev Reflections in Al/n-GaAs/Al Superconductor-Semiconductor-Superconductor Junctions
}

\author{
J. Kutchinsky, ${ }^{1}$ R. Taboryski, ${ }^{2}$ T. Clausen, ${ }^{2}$ C. B. Sørensen, ${ }^{1}$ A. Kristensen, ${ }^{1}$ P. E. Lindelof,,${ }^{1}$ J. Bindslev Hansen, ${ }^{2}$ \\ C. Schelde Jacobsen, ${ }^{2}$ and J. L. Skov ${ }^{2}$ \\ ${ }^{1}$ Niels Bohr Institute, University of Copenhagen, Universitetsparken 5, DK-2100 Copenhagen Ø, Denmark \\ ${ }^{2}$ Department of Physics, Technical University of Denmark, Building 309, DK-2800 Lyngby, Denmark
}

(Received 3 September 1996)

In a highly doped GaAs semiconductor with superconducting contacts of $\mathrm{Al}$, clear conductance peaks are observed at zero voltage bias and at $V= \pm 2 \Delta / e, \pm \Delta / e$. The subharmonic energy gap structure originates from Andreev scattering with diffusive, but energy conserving, transport in the GaAs. The zero bias excess conductance is due to phase-coherent transport. Both effects are suppressed when the distance between the superconducting electrodes exceeds the inelastic diffusion length in the GaAs normal channel. [S0031-9007(96)02273-9]

PACS numbers: 74.50.+r, 73.40.-c, 74.80.Fp

The electronic coupling between two superconductors separated by a mesoscopic normal conductor can manifest itself as a Josephson pair current and/or as a purely resistive correction to the conductance. This latter type of coupling is a recently discovered phenomenon associated with corrections to the well-known proximity effect in normal metals in contact with superconductors, and it has gained considerable interest over the past five years [1-7]. On a microscopic level the proximity effect can be described in terms of Andreev reflections at the S$\mathrm{N}$ interface. The Andreev reflection is a second order process by which an electronlike particle incident on the superconductor with a quasiparticle excitation energy $E$ above the electrochemical potential may be transmitted as part of a Cooper pair if a holelike particle with energy $-E$ is retroreflected along the path of the incoming particle (see top of Fig. 1) [8]. If the excitation energy $E$ is small, the electron and the hole wave packets will be phase coherent but shifted in phase relative to each other by the macroscopic phase of the superconductor $\phi_{1}$ (or $\phi_{2}$ ). The resistive type of coherent coupling between two superconductors separated by a normal conductor relies strongly on the phase coherence of the time reversed paths of the Andreev retroreflected electron-hole pairs $[9,10]$. For such a system after a propagation time $t$ the accumulated phase difference between the electron and the hole wave packets is

$$
\delta \phi=\frac{2 E}{\hbar} t+\frac{2 e}{\hbar} \Phi+\phi_{1}-\phi_{2},
$$

which also includes phase winding by the magnetic flux $\Phi$ enclosed by the path. In the time $t$ the particles will, on the average, diffuse a distance $L=\sqrt{D t}$ in the conductor. Here $D=\frac{1}{3} \nu_{F} \ell_{0}$ is the diffusion constant for diffusion in three dimensions in a conductor with mean free path $\ell_{0}$ and Fermi velocity $\nu_{F}$. This defines the relevant energy scale for complete dephasing of the electron-hole pair: $E_{c}=h D / 2 L^{2}$. Twice this energy $2 E_{c}$ is also referred to as the Thouless energy, which defines a length scale $L_{c}=\sqrt{h D / 2 E_{c}}$. For small excitation energies $L_{c}$ may, by far, exceed both the induced coherence length $\xi_{N}$ in the normal conductor and the size of the normal region. In diffusive normal conductors, $\xi_{N}=\sqrt{\hbar D / 2 \pi k_{B} T}$ is the decay length for the pair amplitude. Inelastic (phasebreaking) scattering or finite sample size will, however, provide a cutoff for the electron-hole coherence. At low temperature the inelastic scattering events in GaAs are dominated by electron-electron interaction with $\ell_{\phi} \gg \xi_{N}$ [11]. Here $\ell_{\phi}=\sqrt{D \tau_{\phi}}$ is the inelastic diffusion length and $\tau_{\phi}$ is the inelastic scattering time. The resistive effect may, therefore, show up on a much longer length scale than the well-known proximity induced Josephson coupling. In contrast to the static proximity effect, this
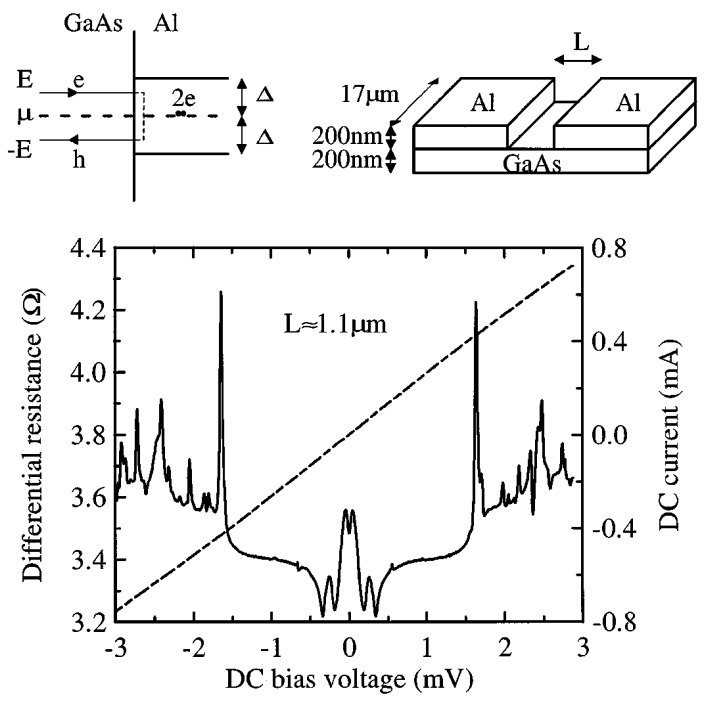

FIG. 1. Differential resistance vs dc bias voltage (solid curve) and $I-V$ characteristic (dashed curve) at $T=0.3 \mathrm{~K}$. Beyond a certain voltage $(\approx \pm 1.6 \mathrm{mV})$, Joule heating drives the superconducting electrodes normal. The structure discussed in the text is seen for $|V|<0.4 \mathrm{mV}$. The measurement shown here was taken for a sample with $L \approx 1.1 \mu \mathrm{m}$. The figures on top show a graphical representation of an Andreev reflection at an NS boundary and the geometry of the SSmS structure. 
new effect is a dynamic (nonequilibrium) phenomenon, which is caused by the perturbation of the electric fields in the normal conductor in close proximity to the superconductor, when a current is passed through the junction $[9,10]$. There is, however, some debate about the interpretation of observations in terms of either the resistive proximity effect mentioned above or a so-called interference model, in which the Andreev reflections only play a role in phase shifting the reflected quasiparticles. In the interference model, resistance osculations with amplitude $\Delta R_{0} \approx R_{0}^{2} e^{2} / h$ are expected due to interfering Feynmann paths [3]. Here $R_{0}$ is the resistance per square and $e^{2} / h$ is the quantum conductance. In some experiments the observed resistance oscillations are, however, up to 2 orders of magnitude larger in amplitude than the theoretical predictions based on the interference model [5,7].

In a superconductor-normal-metal-superconductor (SNS) structure with high transparency of the interfaces there is a high probability for multiple Andreev reflections, where the retroreflected electrons and holes traverse the $N$ region several times. In the $d V / d l$ vs $V$ curves this effect gives rise to the so-called subharmonic energy gap structure (SGS) at dc bias voltages $V= \pm 2 \Delta / n e$, with $n=1,2,3 \ldots$, which is the condition for maximum electron transfer given the number of traversals [12]. The SGS has mostly been observed in Josephson point contacts and microbridges [13] and in very thin semiconductor membranes [14]. The SGS requires conservation of energy for the carriers during traversals of the normal region. Apart from the effect of an applied magnetic field, inelastic scattering provides the only mechanism to randomize the phase and phase-coherent phenomena are thus expected to coexist with the SGS. We have measured the differential resistance in SSmS (Sm: degenerate semiconductor) structures with diffusive Sm channels and observed SGS for long structures with $L \gg \xi_{N}, \ell_{0}$. Even at the lowest accessible temperature, $T=300 \mathrm{mK}$ in the present experiment, our samples were completely resistive. However, an excess conductance corresponding to a dip in the differential resistance was observed at zero voltage bias. From independent weak localization measurements in the GaAs we have determined $\ell_{\phi}$. By varying $L$ we find for the first time direct evidence that both this zero bias dip and the SGS are quenched when the distance between the superconducting electrodes exceeds $\ell_{\phi}$. These phenomena are thus true mesoscopic in the sense that they are observed on a length scale $L$ obeying $\ell_{0} \ll L \leq \ell_{\phi}$. Moreover, the SGS and the zero bias dip exhibit the same temperature dependence, indicating that the two phenomena are related.

Our samples consisted of a $200 \mathrm{~nm}$ heavily doped (degenerate) GaAs layer grown by molecular beam epitaxy (MBE) on an insulating substrate. The GaAs is capped in situ (without breaking the vacuum) with $200 \mathrm{~nm} \mathrm{Al}$. The in situ $\mathrm{Al}$ deposition gives a very smooth and clean interface, which may be crucial for the observation of the
SGS. In order to increase the transparency of the $\mathrm{Al} / \mathrm{GaAs}$ interface five $\delta$-doped layers were incorporated into the GaAs under the Al cap layer. A $17 \mu \mathrm{m}$ wide Hall bar mesa pattern was etched in the $\mathrm{Al} / \mathrm{GaAs}$ structure. Between the voltage probes of the Hall bar a narrow line was drawn across the mesa by electron beam lithography, and the Al film was etched away in a stripe of width $L$. The resulting geometry of the SSmS region is shown in Fig. 1(top). The distance between the voltage probes (not shown in the figure) was $\approx 100 \mu \mathrm{m}$. The fabrication details are given in Ref. [15]. On other samples cut from the same MBE grown wafer the Al film was removed for the assessment of the GaAs conductive layer and for the weak localization measurements. The low temperature mobility of the GaAs conductive layer was $\mu=0.13 \mathrm{~m}^{2} / \mathrm{V} \mathrm{s}$. The carrier density was $n_{e}=4.8 \times 10^{24} \mathrm{~m}^{-3}$, corresponding to a mean free path of $\ell_{0} \approx 50 \mathrm{~nm}$ and a diffusion constant $D=0.016 \mathrm{~m}^{2} / \mathrm{s}$. The Al film had a critical temperature close to the bulk value $T_{c}=1.2 \mathrm{~K}$, and the bulk value for the superconducting energy gap $\Delta(0) / e \approx 175 \mu \mathrm{V}$ was used. By using the transmission line method [16], we determined the specific contact resistivity in the normal state to be $\rho_{N}=53 \times 10^{-12} \Omega \mathrm{m}^{2}$. At each $\mathrm{Al} / \mathrm{GaAs}$ interface the current will flow from the highly conductive Al film to the more resistive GaAs over a decay length $\ell_{N}=\sqrt{d \rho_{N} / \rho_{\mathrm{GaAs}}} \approx 0.9 \mu \mathrm{m}$, where $d$ and $\rho_{\mathrm{GaAs}}$ are the thickness and the resistivity of the conductive GaAs layer, respectively. An estimate of the barrier transmission coefficient $T$ based on the excess current at high bias gave $T_{n} \approx 0.5$, corresponding to a $Z$ factor of order 1 . The $Z$ factor is the normalized interface barrier height as defined in Ref. 17.

The measurements were carried out with the use of a phase sensitive detection technique that allowed us to measure the dc $I-V$ characteristics and the differential resistance $d V / d l$ vs $V$ simultaneously. In the differential measurement the ac voltage level was kept well below the thermal energy $e \delta V_{\mathrm{rms}} \ll k_{B} T$ in order to avoid smearing of the structure observed in the differential resistance. Most of the measurements were performed in a conventional pumped ${ }^{3} \mathrm{He}$ cryostat with a base temperature of about $300 \mathrm{mK}$. In Fig. 1 we show an example of the measurements. The $I-V$ characteristic (shown as the dashed line) exhibits only weak structure, although, in principle, it contains the same information as the differential resistance (solid line). At a certain sample dependent voltage $V_{c}(\approx \pm 1.6 \mathrm{mV}$ in Fig. 1) Joule heating caused a breakdown of superconductivity in the Al electrodes and drove the resistance to the normal state level superimposed by irregular structure. The high peaks at $\pm V_{c}$ are caused by a breakdown of a small excess current observed in the superconducting state. $V_{c}$ has a temperature dependence given by $\sqrt{T_{c}-T}$ corresponding to an energy balance condition, where the dissipated power $P=V^{2} / R$ is proportional to the temperature shift [15]. Well below $V_{c}$ we observed a pronounced and fully symmetric structure in 


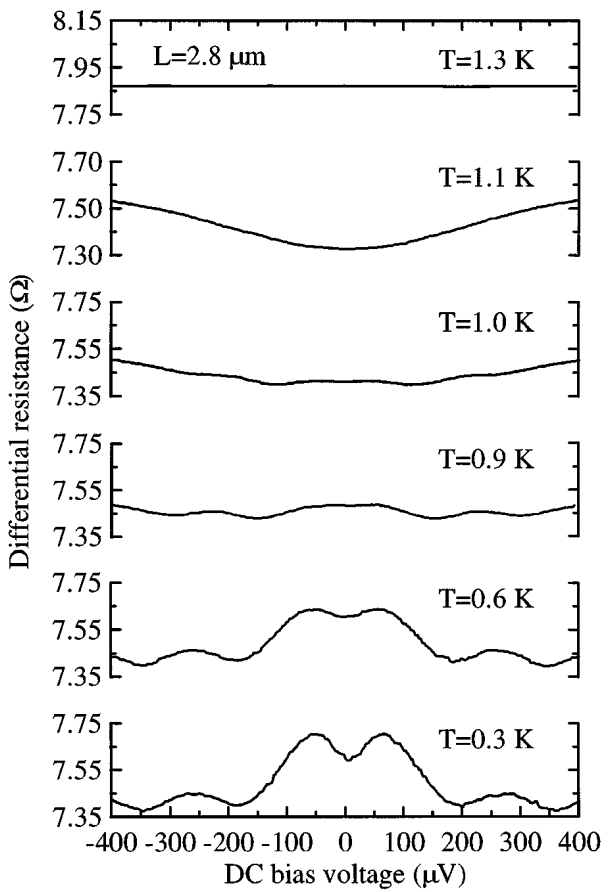

FIG. 2. Temperature dependence of the differential resistance vs dc bias voltage for a typical sample with $L \approx 2.8 \mu \mathrm{m}$. Above $T_{c} \approx 1.2 \mathrm{~K}$ the structure disappears.

the differential resistance. This structure consists of dips at $V= \pm 2 \Delta / e, \pm \Delta / e$, and a "saddle" shaped structure around zero bias. As seen in Fig. 2 all this structure had a strong temperature dependence and disappeared completely when the temperature exceeded the critical temperature. The precise shape of the structure, in particular, the saddle shaped rise in differential resistance, is not understood and calls for further theoretical investigation. The dip in the middle of the saddle is the so-called excess conductance also observed by others [1,2]. It is known to be a signature of the phase-coherent resistive coupling between the two superconducting electrodes. In Ref. [18] an effective suppression voltage for the excess conductance peak is estimated to equal some fraction of $\delta V_{c} \approx h \nu_{F} / 2 e \ell_{0}$. For our sample parameters $\delta V_{c} \approx 39 \mathrm{mV}$ which seems to be in rather poor agreement with the present experiment. The FWHM of this dip appears to match the thermal energy $k_{B} T / e(=22 \mu \mathrm{V}$ at $T=0.3 \mathrm{~K})$ much better. The dips at $V= \pm 2 \Delta / e, \pm \Delta / e$ are the SGS originating from Andreev reflections. In order to find the relevant length scale for the observed structures, we fabricated samples on the same GaAs wafer with different lengths $L$ of the Sm region. The results are seen in Fig. 3, which presents the key novel observation of this Letter. Here we show the differential resistance for samples with varying $L$. It is evident that the zero bias dip and the SGS fade out with increasing $L$ and become completely absent for $L>3.5 \mu \mathrm{m}$. For comparison, the theoretical value of the coherence length at $0.3 \mathrm{~K}$ is $\xi_{N}=250 \mathrm{~nm}$. We would like to emphasize that

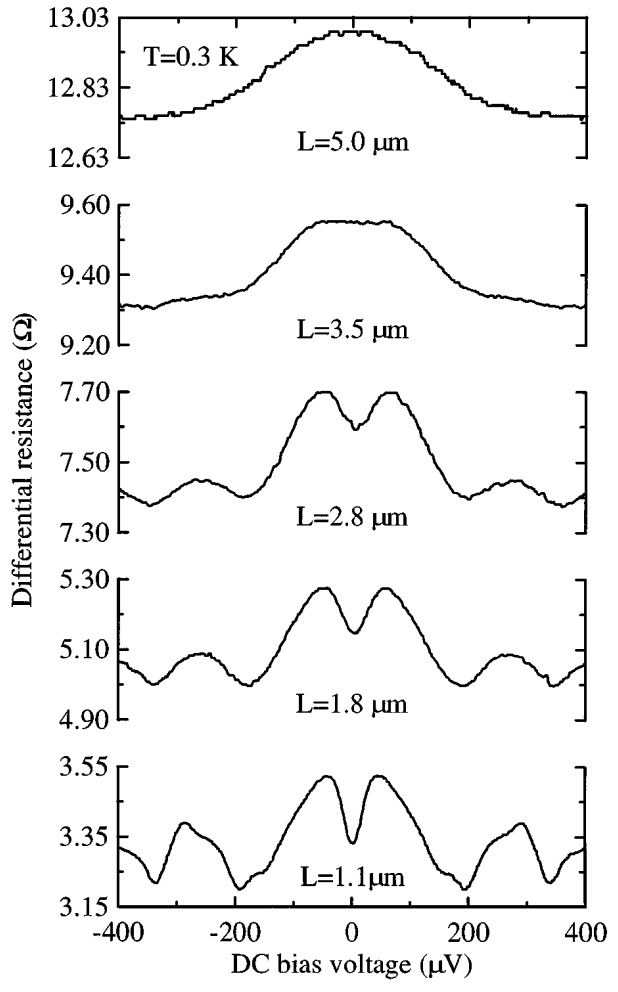

FIG. 3. The differential resistance vs dc bias voltage for samples with varying $L$. All the measurements are taken at $T \approx 300 \mathrm{mK}$. For $L \geq 3.5 \mu \mathrm{m}$ both the zero bias dip and the SGS are suppressed.

the positions in dc bias voltage of the SGS dips are exactly the same in all well-cooled samples with $L \leq 3.5 \mu \mathrm{m}$ as expected for multiple Andreev reflections. In Ref. [15] this point was masked by the self-heating effect mentioned earlier. Characteristic features in the $d V / d l$ vs $V$ curves that only depend on decoupled SN interfaces will scale in bias position with the voltage drop $R_{\mathrm{GaAs}} I$ across the normal region, where $R_{\mathrm{GaAs}}$ is the resistance of the normal GaAs region of length $L$. When increasing $L$ the saddle shaped increase in differential resistance near zero bias is hardly affected. However, it has a strong temperature dependence (see Fig. 3). We thus believe this feature is an independent property of each S-Sm interface. The question now arises if the measured (surprisingly long) decay length $L_{\max }$ for the coherent coupling between the two superconductors can be related to the inelastic diffusion length $\ell_{\phi}$ in the $\mathrm{Sm}$ region. In order to check this we prepared samples cut from the same MBE grown wafer but with the Al top layer removed from the area between the contacts of a Hall bar. For these samples we measured the low field magnetoresistance of the GaAs conductive layer and found the weak localization contribution to the magnetoresistance. Weak localization is a quantum mechanical correction to classical conductivity, which arises from the phase coherence of self-intersecting time reversed trajectories. This phase coherence is gradually destroyed when a 
magnetic field is applied. This is seen as a definite feature in the magnetoresistance. In Ref. [19] the weak localization magnetoresistance is given as

$$
\begin{aligned}
\frac{\Delta R(B)}{R}=-\frac{1}{R_{0}} \frac{e^{2}}{2 \pi^{2} \hbar}\{ & \Psi\left(\frac{1}{2}+\frac{B_{1}}{B}\right)-\frac{3}{2} \Psi\left(\frac{1}{2}+\frac{B_{2}}{B}\right) \\
& \left.+\frac{1}{2} \Psi\left(\frac{1}{2}+\frac{B_{3}}{B}\right)\right\},
\end{aligned}
$$

where $\Psi(x)$ is the digamma function. Neglecting spin-flip processes, but retaining spin-orbit scattering, the fitting parameters are given by the characteristic fields $B_{1}=$ $B_{0}+B_{\text {so }}, B_{2}=4 / 3 B_{\text {so }}+B_{\phi}$, and $B_{3}=B_{\phi}$, with $B_{0}=$ $\hbar \nu_{F} / 4 e D \ell_{0}, B_{\mathrm{so}}=\hbar / 4 e \ell_{\mathrm{so}}^{2}$, and $B_{\phi}=\hbar / 4 e \ell_{\phi}^{2}$. $\ell_{\mathrm{so}}$ is the spin-orbit diffusion length in analogy with $\ell_{\phi}$. In the fitting procedure the mean free path $\ell_{0}$ is known from transport measurements. The presence of spin-orbit scattering in $\mathrm{GaAs}$ is due to the spin splitting of the band structure [20]. $\ell_{\phi}$ is the interesting quantity here and the only one that has a significant temperature dependence. In Fig. 4 we show the magnetoresistance with a fit to Eq. (2) and the determination of the inelastic diffusion length $\ell_{\phi}$. At $0.3 \mathrm{~K} \ell_{\phi} \approx 2.8 \mu \mathrm{m}$, which is in excellent agreement with the observed decay length $L_{\max } \approx 3.5 \mu \mathrm{m}$ for the zero-bias dip and for the SGS in the $d V / d l$ vs $V$ curves of our SSmS samples. As expected $L_{\max }$ decreases when the temperature is raised. However, a functional dependence cannot be extracted from the data.

In conclusion, we have shown that the decay length for two characteristic features in the $d V / d l$ vs $V$ curves

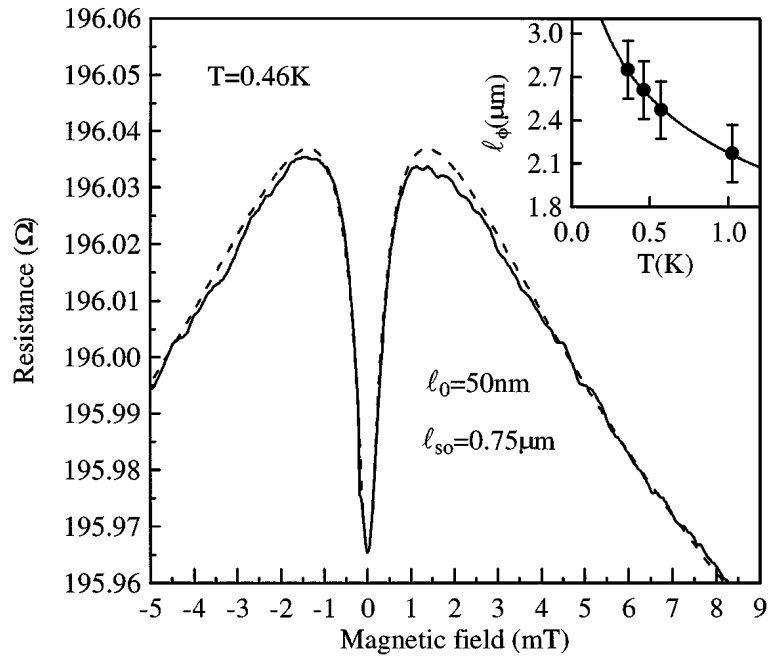

FIG. 4. Weak localization magnetoresistance of GaAs for a sample where the Al top layer has been removed $(T=0.46 \mathrm{~K})$. The sharp dip at zero field is the positive magnetoresistance (weak antilocalization) caused by the spin-orbit coupling in GaAs. The spin-orbit coupling introduces an extra fitting parameter $\ell_{\mathrm{so}}$. The dashed curve is a fit to the data using the expression in Eq. (2). The fitting parameters are shown in the figure and in the inset as a function of temperature. The mean free path $\ell_{0}$ was obtained independently from transport measurements. for planar superconductor-semiconductor-superconductor junctions is the inelastic diffusion length $\ell_{\phi}(\approx 2.8 \mu \mathrm{m}$ at $0.3 \mathrm{~K}$ ), which we have determined independently by weak localization measurements on the diffusive semiconductor material. The zero bias excess conductance is a signature of a resistive but phase-coherent correction to the proximity coupling between the two superconductors which persists over length scales much longer than the coherence length $\xi_{N}(=250 \mathrm{~nm}$ at $0.3 \mathrm{~K})$ for the pair amplitude. This type of coherent coupling is cut off at a distance given by $\ell_{\phi}$, which also sets the cutoff length for the subharmonic energy gap structure (SGS). The similar length and temperature dependence of the two phenomena indicate a relation between them which calls for further studies.

We acknowledge useful discussions with Dr. Hideaki Takayanagi, Dr. Junsaku Nitta, Professor Henrik Smith, and Professor Teun M. Klapwijk. This work was supported by the Danish Technical Research Council. We also thank CNAST for support and the III-V Nanolab at the Niels Bohr Institute for providing us with processing facilities.

[1] A. Kastalsky et al., Phys. Rev. Lett. 67, 3026 (1991).

[2] Chang Nguyen, H. Kroemer, and E.L. Hu, Phys. Rev. Lett. 69, 2847 (1992).

[3] P. G. N. de Vegvar et al., Phys. Rev. Lett. 73, 1416 (1994).

[4] H. Pothier et al., Phys. Rev. Lett. 73, 2488 (1994).

[5] V. T. Petrashov et al., Phys. Rev. Lett. 74, 5268 (1995).

[6] A. Dimoulas et al., Phys. Rev. Lett. 74, 602 (1995).

[7] H. Courtois et al., Phys. Rev. Lett. 76, 130 (1996).

[8] A. F. Andreev, Zh. Eksp. Teor. Fiz. 46, 1823 (1964) [JETP 19, 1228 (1964)].

[9] Fei Zhou, B. Spivak, and A. Zyuzin, Phys. Rev. B 52, 4467 (1995).

[10] A. Volkov and H. Takayanagi, Phys. Rev. Lett. 76, 4026 (1996).

[11] R. Taboryski and P.E. Lindelof, Semicond. Sci. Technol. 5, 933 (1990).

[12] M. Octavio, M. Tinkham, G.E. Blonder, and T.M. Klapwijk, Phys. Rev. B 27, 6739 (1983); K. Flensberg, J. Bindslev Hansen, and M. Octavio, Phys. Rev. B 38, 8707 (1988).

[13] P.E. Gregers-Hansen et al., Phys. Rev. Lett. 31, 524 (1973).

[14] W. M. van Huffelen et al., Phys. Rev. B 47, 5170 (1993).

[15] R. Taboryski et al., Appl. Phys. Lett. 69, 656 (1996).

[16] H. K. Henish, Semiconductor Contacts (Oxford University Press, New York, 1989), pp. 14-18.

[17] G. E. Blonder, M. Tinkham, and T. M. Klapwijk, Phys. Rev. B 25, 4515 (1982).

[18] B. J. van Wees et al., Phys. Rev. Lett. 69, 510 (1992).

[19] S. Hikami, A. I. Larkin, and Y. Nagaoka, Prog. Theor. Phys. 63, 707 (1980).

[20] W. Knap et al., Phys. Rev. B 53, 3912 (1996). 